\title{
Prenatal Stress due to a Natural Disaster Predicts Adiposity in Childhood: The Iowa Flood Study
}

\author{
Kelsey N. Dancause, ${ }^{1}$ David P. Laplante, ${ }^{2}$ Kimberly J. Hart, ${ }^{3}$ Michael W. O'Hara, ${ }^{4}$ \\ Guillaume Elgbeili, ${ }^{2}$ Alain Brunet, ${ }^{2,5}$ and Suzanne King ${ }^{2,5}$ \\ ${ }^{1}$ Université du Québec à Montréal (UQAM), Montreal, QC, Canada H2X $1 Y 4$ \\ ${ }^{2}$ Douglas Hospital Research Centre, Montreal, QC, Canada H4H 1 R3 \\ ${ }^{3}$ University of Illinois at Chicago, Chicago, IL 60607, USA \\ ${ }^{4}$ University of Iowa, Iowa City, IA 52242, USA \\ ${ }^{5}$ McGill University, Montreal, QC, Canada H3A 0G4
}

Correspondence should be addressed to Suzanne King; suzanne.king@mcgill.ca

Received 28 July 2014; Revised 27 November 2014; Accepted 11 December 2014

Academic Editor: Li Ming Wen

Copyright (C) 2015 Kelsey N. Dancause et al. This is an open access article distributed under the Creative Commons Attribution License, which permits unrestricted use, distribution, and reproduction in any medium, provided the original work is properly cited.

Prenatal stress can affect lifelong physical growth, including increased obesity risk. However, human studies remain limited. Natural disasters provide models of independent stressors unrelated to confounding maternal characteristics. We assessed degree of objective hardship and subjective distress in women pregnant during severe flooding. At ages 2.5 and 4 years we assessed body mass index (BMI), subscapular plus triceps skinfolds (SS + TR, an index of total adiposity), and SS : TR ratio (an index of central adiposity) in their children $(n=106)$. Hierarchical regressions controlled first for several potential confounds. Controlling for these, flood exposure during early gestation predicted greater BMI increase from age 2.5 to 4 , as well as total adiposity at 2.5. Greater maternal hardship and distress due to the floods, as well as other nonflood life events during pregnancy, independently predicted greater increase in total adiposity between 2.5 and 4 years. These results support the hypothesis that prenatal stress increases adiposity beginning in childhood and suggest that early gestation is a sensitive period. Results further highlight the additive effects of maternal objective and subjective stress, life events, and depression, emphasizing the importance of continued studies on multiple, detailed measures of maternal mental health and experience in pregnancy and child growth.

\section{Introduction}

Researchers and public health officials have long recognized the role of maternal health during pregnancy in shaping the health of the infant. In the last three decades, research in the developmental origins of health and disease has highlighted effects extending well beyond infancy $[1,2]$. Children whose mothers had poor nutrition during pregnancy are more likely to be born small and have greater risk for obesity and diabetes, particularly if they have rapid growth in the first weeks [3] or months $[4,5]$ of life. In addition to the prenatal nutritional environment, prenatal stress is increasingly recognized to contribute to cardiometabolic disease risk [6], including later obesity [7] and features of diabetes $[8,9]$. This likely reflects effects of maternal stress hormones which, at high levels, can cross the placental barrier and affect fetal development [10], as well as epigenetic changes in the placenta and fetus [1113]. In addition to adverse effects on fetal growth, which is an independent risk factor for obesity [14], maternal stress might influence long-term metabolic outcomes through effects on the developing hypothalamic pituitary adrenal axis $[12,15]$ or on metabolism at the cellular level [16] and thereby increase obesity risk independent of effects on birth weight [17].

Despite this growing body of evidence, studies of the effects of prenatal stress on physical growth in humans remain limited. Danish National Register studies indicated that bereavement due to death of a close relative during or shortly before pregnancy was associated with increased risk of overweight among the women's children from ages 10 to 13 years [18] and in early adulthood [19]. Similarly, results 
from Project Viva, a prospective cohort study of pregnant women and their children, indicated smaller body size but greater central adiposity at age 3 years in association with antenatal depression [20] and with 2nd trimester maternal corticotropin-releasing hormone $(\mathrm{CRH})$, which provides a marker of fetal glucocorticoid exposure [21]. However, a Danish National Birth Cohort study examining a combined measure of maternal distress in pregnancy, reflecting selfreported anxious, depressive, or stress symptoms, showed no associations with offspring overweight at age 7 [22]. These results highlight some of the difficulties of designing human studies of prenatal stress: effects might differ for stress, anxiety, depression, or hormonal markers of stress. Furthermore, anxiety, depression, and bereavement might be associated with one another and with other maternal characteristics that can influence child development. Finally, the effects of prenatal stress on later growth and development depend on the timing of exposure during gestation [23, 24], but many human studies are not able to evaluate the timing of the stressor with accuracy. Thus, we need more studies examining the effects of independent stressors during pregnancy on body composition in children.

Natural disasters provide excellent opportunities to examine the effects of prenatal stress on childhood outcomes because the stressors are independent of potentially confounding genetic and medical risk factors and are relatively randomly distributed with regard to household and maternal characteristics. Furthermore, because the dates of the events are clearly known, we can identify the timing of stress exposure during pregnancy [25]. Our first prospective longitudinal study of prenatal stress due to a natural disaster, Project Ice Storm, has followed the development of children whose mothers were pregnant during a severe ice storm in 1998. Exposure to the ice storm was associated with shorter length at birth [26] and with measures of physical growth later in childhood. Greater objective hardship due to the storm predicted greater body mass index (BMI) and increased risk of obesity at age 5.5 [27], as well as insulin secretion and BMI in adolescence [28]. However, this study left unanswered questions about the effects of prenatal stress on physical growth in early childhood.

In June 2008, an opportunity to replicate Project Ice Storm arose when the U.S. Midwest experienced its worst flooding in more than 50 years. We recruited women exposed to the floods during pregnancy, assessed their stress levels soon after the floods, and collected anthropometric measurements among their children at ages 2.5 and 4 years. We examined relationships between timing and severity of flood exposure and these body composition measurements.

\section{Materials and Methods}

All phases of this study were approved by the University of Iowa Institutional Review Board.

2.1. Participants. Immediately following the start of the flooding, we recontacted women enrolled in an existing study of maternal characteristics and pregnancy outcomes at the University of Iowa [29], who had initially been recruited at $<20$ weeks of gestation from the University of Iowa Hospitals and Clinics. We recruited additional women from three severely flood-affected counties (Linn, Johnson, and Blackhawk). All women were of age 18 or older and English speaking. Of 323 women approached, 268 provided information concerning the flood; 217 were pregnant at the time of the floods.

Families were invited to participate in assessments of children's behavioral, cognitive, and physical outcomes when children were 2.5 and 4 years of age. At age 2.5, 131 families participated in assessments: 27 completed only postal questionnaires and 104 completed face-to-face assessments, when anthropometric measurements were collected. At age 4, 105 families participated in assessments: 24 completed only postal questionnaires and 81 completed face-to-face assessments.

Anthropometric data were missing for some participants, leaving a final sample of 106 women who were exposed to the floods in the 3rd $(n=34)$, 2nd $(n=41)$, or 1st $(n=31)$ trimester and their children (58 boys, 48 girls) who participated in the assessments at age 2.5 only $(n=29)$, age 4 only $(n=7)$, or both ages $(n=70)$.

2.2. Assessments. Anthropometric measurements were collected following standard guidelines [30]. Standing height was measured without shoes to the nearest $0.1 \mathrm{~cm}$ and weight to the nearest $0.1 \mathrm{~kg}$ for mothers and children. Children's triceps and subscapular skinfolds were measured three times each on the right side of the body using Lange calipers, and the mean of the three measurements was used for analyses.

2.3. Control Variables. At recruitment in 2008, we collected demographic information; maternal medical and obstetric history; and information on smoking (number of cigarettes/day) and alcohol consumption (number of drinks/ week) during pregnancy, using telephone interviews and mail questionnaires. Socioeconomic status (SES) was determined based on parental education and occupation status using the Hollingshead Social Position Criteria [31]. Medical and obstetric history variables relevant to the tested outcomes were combined into an obstetric/fetal risk factor variable, which included history of kidney disease; hypertension; anemia; heart disease; seizures; diabetes; HIV; Rh negative status; asthma; sexually transmitted infections; abnormal blood clotting; thyroid disorders; vaginal, cervical, or urinary tract infections; endocrine disorders; abnormal pregnancy weight gain $(<4 \mathrm{~kg}$ or $>18 \mathrm{~kg})$; preeclampsia; or abnormal bleeding during pregnancy.

Twelve months after the flood, we assessed stressful maternal life events (other than the flood) using the Life Experiences Survey (LES) [32], a self-report measure of life changes, such as death of a spouse or a work promotion. Women were instructed to indicate events occurring from the beginning of their 2008 pregnancy up to the present day. Mothers also completed the Inventory of Depression and Anxiety Symptoms (IDAS) [33], a self-report measure of depression and anxiety symptoms. 
At the 2.5-year assessments, we collected data on breastfeeding patterns using semistructured interviews, during which women recalled the age and duration of exclusive, predominant, and mixed breastfeeding, as well as the age of introduction of other foods.

\subsection{Flood-Related Variables}

2.4.1. Objective Hardship. We assessed the severity of floodrelated events experienced by participants using a questionnaire that tapped into four categories used in other disaster studies: Threat, Loss, Scope, and Change [34]. Because each natural disaster presents unique experiences, questions must be tailor-made. Our scale included questions specific to the flood, such as days without electricity, damage to the home, and danger due to flood waters. Each dimension was scored on a scale of 0-25 ranging from no exposure to high exposure. A total score (IF100) was calculated by summing the four dimensions using McFarlane's approach [35]. A detailed presentation of the scale is presented elsewhere [36]. In the present sample, scores ranged from 0 to 50 out of a possible 100 points.

2.4.2. Subjective Distress. We assessed women's psychological reaction to the flood using the Impact of Event Scale-Revised (IES-R) [37]. This 22-item scale describes symptoms from 3 categories relevant to posttraumatic stress disorder: intrusions (thoughts and images), hyperarousal, and avoidance. Participants responded on a 5-point Likert scale, from " 0 Not at all" to "4-Extremely," the extent to which each behavior described how they felt over the preceding 7 days. Items were written to reflect symptoms relative to the flood. The total score was used in analyses. In the present sample, scores ranged from 0 to 60 out of a possible 88 points.

2.4.3. Timing of Exposure. The timing of flood exposure during pregnancy was defined as the number of days between June 15, 2008-the peak of the floods-and the infant's due date. Third trimester exposure corresponds to due dates falling between 0 and 93 days following June 15th; 2nd trimester, 94-186 days; and 1st trimester, 187-279 days.

2.5. Outcome Variables. Outcome variables included sexand age-specific body mass index $Z$-scores based on Center for Disease Control (CDC) child growth standards [38]; subscapular and triceps skinfold sum (SS + TR), an index of total adiposity [39]; and subscapular to triceps skinfold ratio (SS : TR), an index of central adiposity [39].

2.6. Statistical Analyses. Objective hardship (IF100) and subjective distress (IES-R) scores were right-skewed and were thus log-transformed for analysis. In addition to child sex, eight covariates expected to be potentially related to child outcomes based on the literature reviews were included in analyses: birth weight (g), obstetric/fetal risk score, maternal BMI (measured during the 2.5-year assessments for analyses of child outcomes at age 2.5 and for the difference between ages 2.5 and 4 and measured during the 4 -year assessments for analyses of child outcomes at age 4), smoking during pregnancy (per day), breastfeeding duration (months), SES, general depression, and number of life events.

We tested relationships between predictor and outcome variables using hierarchical linear regression. In a series of individual steps, we first entered child sex and control variables, followed by flood variables: exposure timing, objective hardship, and subjective distress. In a second set of models, for analyses of SS + TR and SS : TR, we included child BMI $Z$-score in the control variables, measured during the 2.5year assessments for analyses at age 2.5 and for the difference between ages 2.5 and 4 and measured during the 4-year assessments for analyses at age 4. Finally, in a third set of models, we entered interactions after the flood variables, including objective hardship $*$ sex, subjective distress $*$ sex, objective hardship $*$ timing, subject distress $*$ timing, and objective hardship $*$ subjective distress. All tests used an a priori alpha level of 0.05 (two-sided tests). No measure was taken to correct for multiple testing, as analyses were considered exploratory. Analyses were conducted with SPSS 20.0.

\section{Results}

3.1. Sample Characteristics and Correlations. Table 1 presents correlations among all study variables as well as their means and standard deviations. Significant correlations suggested that greater objective hardship (IF100) predicted a greater increase in total adiposity between ages 2.5 and 4 . Greater subjective maternal distress (IES-R) predicted greater total adiposity at both 2.5 and 4 years. Timing of the floods earlier in gestation predicted greater BMI at age 4, a greater increase in BMI between the two assessments, and greater total adiposity at ages 2.5 and 4 years. Several control variables were also significantly correlated with outcomes. There were no mean differences in predictor variables (flood variables or covariates) among participants who were measured at only age 2.5 , only age 4 , or both ages (data not shown).

3.2. Multiple Linear Regression Models. Results of regression analyses for each outcome variable are shown in Tables 24 and show the progression of variance explained $\left(R^{2}\right)$ with each step.

\subsection{Body Mass Index (BMI) Z-Scores (Table 2)}

3.3.1. Age 2.5. At entry into the model, birth weight $(P=$ $0.03)$ and maternal BMI $(P=0.01)$ predicted child BMI $Z$ scores. In the final model, larger birth weight $(P=0.03)$, fewer fetal risk factors $(P=0.01)$, and larger maternal BMI $(P=0.03)$ predicted greater BMI $Z$-scores. There were no effects of severity of objective hardship or subjective distress due to flood exposure nor of the timing of flood exposure.

3.3.2. Age 4. At entry into the model, fetal risk factors $(P=$ $0.04)$ and maternal BMI $(P<0.01)$ predicted child BMI $Z$ scores. In the final model, fetal risk factors did not retain significance $(P=0.56)$. Larger maternal BMI $(P<0.01)$ 
TABLE 1: Correlations among predictor and outcome variables and descriptive statistics.

\begin{tabular}{|c|c|c|c|c|c|c|c|c|c|c|c|c|c|c|c|}
\hline & 1 & 2 & 3 & 4 & 5 & 6 & 7 & 8 & 9 & 10 & 11 & 12 & Mean & SD & $n$ \\
\hline \multicolumn{16}{|l|}{ Predictors } \\
\hline 1 Obj. hardship & 1 & $40^{* *}$ & 0.18 & 0.05 & -0.15 & 0.09 & -0.01 & 0.09 & -0.06 & -0.09 & 0.18 & $.22^{*}$ & 1.8 & 0.8 & 106 \\
\hline 2 Subj. distress & $0.40^{* *}$ & 1 & 0.00 & 0.10 & -0.02 & 12 & 0.02 & -0.01 & $-0.26^{*}$ & $-0.21^{*}$ & $0.30^{* *}$ & 0.19 & 1.3 & 1.1 & 106 \\
\hline 3 Timing & 0.18 & 0.00 & 1 & 0.07 & -0.02 & 0.04 & -0.08 & -0.10 & 0.10 & 0.02 & -0.17 & -0.01 & 140.5 & 78.0 & 106 \\
\hline 4 Birth weight & -0.05 & 0.10 & 0.07 & 1 & -0.02 & 0.02 & -0.08 & -0.05 & 0.06 & 0.02 & 0.09 & 0.02 & 3531 & 469 & 106 \\
\hline 5 Fetal risk & -0.15 & -0.02 & -0.02 & -0.02 & 1 & $0.57^{* *}$ & $0.53^{* *}$ & 0.06 & $-0.25^{*}$ & -0.18 & -0.11 & 0.13 & 0.6 & 0.9 & 106 \\
\hline 6 Mat. & 0.09 & 0.12 & 0.04 & 0.02 & $0.57^{* *}$ & 1 & $0.92^{* *}$ & 0.00 & $-0.40^{* *}$ & -0.19 & 0.06 & $0.26^{* *}$ & 26.5 & 5.8 & 99 \\
\hline 7 Mat. BMI 4 & -0.01 & 0.02 & -0.08 & -0.08 & $0.53^{* *}$ & $0.92^{* *}$ & 1 & 0.09 & $-0.46^{* *}$ & -0.20 & 0.02 & $0.27^{*}$ & 27.3 & 7.3 & 75 \\
\hline 8 Smoking & 0.09 & -0.01 & -0.10 & -0.05 & 0.06 & 0.00 & 0.09 & 1 & 0.01 & -0.14 & 0.18 & $0.25^{*}$ & 0.3 & 1.5 & 106 \\
\hline $9 \mathrm{BF}$ duration & -0.06 & $-0.26^{*}$ & 0.10 & 0.06 & $-0.25^{*}$ & $-0.40^{* *}$ & $-0.46^{* *}$ & 0.01 & 1 & $0.26^{*}$ & -0.07 & -0.03 & 8.2 & 7.0 & 98 \\
\hline $10 \mathrm{SES}$ & -0.09 & $-0.21^{*}$ & 0.02 & 0.02 & -0.18 & -0.19 & -0.20 & -0.14 & $0.26^{*}$ & 1 & -0.16 & $-0.26^{* *}$ & 3.3 & 9.8 & 106 \\
\hline Mat. & 0.18 & $0.30^{* *}$ & -0.17 & 0.09 & -0.11 & 0.06 & 0.02 & & -0.07 & -0.16 & 1 & $0.42^{* *}$ & 3.2 & 8.2 & 106 \\
\hline Mat. life events & $0.22^{*}$ & 0.19 & -0.01 & 0.02 & 0.13 & $0.26^{* *}$ & $0.27^{*}$ & $0.25^{*}$ & -0.03 & $-0.26^{* *}$ & $0.42^{* *}$ & 1 & 3.0 & 2.1 & 106 \\
\hline \multicolumn{16}{|l|}{ Child outcomes } \\
\hline BMIZ 2.5 & 0.02 & 0.17 & -0.05 & $0.24^{*}$ & -0.12 & 0.14 & $0.31^{*}$ & 0.03 & -0.09 & -0.09 & 0.08 & -0.01 & -0.03 & 1.00 & 98 \\
\hline BMIZ 4 & -0.15 & 0.08 & $-0.24^{*}$ & 0.16 & 0.20 & $0.45^{* *}$ & $0.51^{* *}$ & -0.03 & -0.19 & -0.12 & 0.07 & 0.14 & 0.40 & 1.09 & 77 \\
\hline BMIZ dif. & 0.06 & 0.01 & $-0.28^{*}$ & -0.08 & $0.34^{* *}$ & $0.29^{*}$ & $0.34^{* *}$ & $0.29^{*}$ & -0 & -0.05 & -0 & 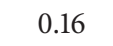 & 0.38 & 0.85 & 69 \\
\hline SS + TR 2.5 & 0.09 & 0.10 & $-0.25^{*}$ & 0.06 & -0.12 & 0.05 & 0.22 & -0.05 & -0.10 & 0.13 & 0.09 & -0.10 & 13.9 & 2.7 & 88 \\
\hline $\mathrm{SS}+\mathrm{TR} 4$ & 0.13 & $0.26^{*}$ & $-0.26^{*}$ & 0.09 & 0.08 & $0.32^{*}$ & $0.42^{* *}$ & 0.15 & -0.24 & -0.22 & 0.04 & 0.14 & 17.2 & 3.7 & 62 \\
\hline SS + TR dif. & $0.38^{* *}$ & $0.42^{* *}$ & -0.18 & 0.07 & 0.19 & $0.28^{*}$ & $0.36^{*}$ & $0.67^{* *}$ & -0.11 & -0.16 & 0.03 & 0.27 & 3.3 & 3.1 & 52 \\
\hline SS : TR 2.5 & 0.03 & -0.06 & & 0.00 & -0.20 & -0.04 & -0.01 & & 0.19 & -0.07 & -0.07 & 0.10 & 0.61 & 0.17 & 88 \\
\hline SS: TR 4 & -0.11 & 0.03 & -0.13 & 0.23 & -0.08 & -0.11 & -0.11 & 0.03 & 0.15 & 0.00 & 0.23 & -0.02 & 0.64 & 0.22 & 62 \\
\hline SS : TR dif. & -0.04 & 0.18 & -0.19 & 0.27 & 0.02 & -0.13 & -0.13 & 0.04 & 0.11 & 0.09 & $0.36^{* *}$ & -0.07 & 0.06 & 0.26 & 52 \\
\hline
\end{tabular}

${ }^{*} P<0.05 ;{ }^{* *} P<0.01$. Abbreviations: Obj. hardship: objective hardship; Subj. distr.: subjective distress; Mat.: maternal; BMI: body mass index; BF duration: breastfeeding duration (months); SES: socioeconomic status; gen. depr.: general depression; BMIZ: body mass index Z-Score; Dif.: difference between values at age 2.5 and 4 years; SS: subscapular skinfold; TR: triceps skinfold.

predicted greater BMI $Z$-scores. There were no effects of the severity or timing of flood exposure.

3.3.3. Difference between Ages 2.5 and 4. At entry into the model, fetal risk factors $(P<0.01)$ and maternal smoking $(P=0.04)$ predicted difference in BMI $Z$-scores. There were no effects of the severity of hardship or distress due to flood exposure. However, earlier timing of exposure $(P=0.04)$ predicted a greater increase in BMI $Z$-scores from age 2.5 to 4 . In the final model, fetal risk factors $(P=0.09)$ and smoking $(P=0.08)$ did not retain significance, but earlier timing of flood exposure $(P=0.03)$ predicted a greater increase in BMI $Z$-scores from age 2.5 to 4 .

\subsection{Total Adiposity $(S S+T R)$ (Table 3)}

3.4.1. Age 2.5. There were no effects of covariates or of the severity of objective hardship or subjective distress due to the flood on total adiposity. However, at entry into the model $(P=0.04)$ and in the final model $(P=0.03)$, exposure timing predicted total adiposity at age 2.5 : earlier timing of exposure predicted greater adiposity. The effects of exposure timing remained significant in the final model $(P=0.03)$ even when controlling for child BMI $Z$-score at age 2.5 (full results not shown).

3.4.2. Age 4. At entry into the model, maternal BMI $(P<$ $0.01)$ and smoking $(P<0.01)$ predicted total adiposity. In the final model, fewer fetal risk factors $(P=0.01)$, larger maternal BMI $(P<0.01)$, and more smoking during pregnancy $(P<$ 0.01 ) predicted greater adiposity. There were no effects of the severity or timing of flood exposure on total adiposity at age 4.

3.4.3. Difference between Ages 2.5 and 4. At entry into the model, smoking $(P<0.01)$ predicted the difference in total adiposity between ages 2.5 and 4 . In addition, the severity of both objective hardship $(P=0.02)$ and subjective distress $(P=0.04)$ due to the floods predicted the difference in adiposity between ages 2.5 and 4 . In the final model, more smoking during pregnancy $(P<0.01)$, a greater number of maternal life events $(P=0.04)$, greater objective hardship due to the flood $(P=0.03)$, and greater subjective distress due to the flood $(P=0.04)$ all predicted a greater increase in total adiposity. The effects of objective hardship and subjective distress remained significant $(P=0.03$ and $P=0.04$, resp. $)$ 
TABLE 2: Summary of hierarchical linear regression analyses for body mass index (BMI). Significant effects are indicated in bold.

\begin{tabular}{|c|c|c|c|c|c|c|c|c|}
\hline \multirow{2}{*}{ Predictor variables } & \multicolumn{5}{|c|}{ Values at entry into the model } & \multicolumn{3}{|c|}{ Final model } \\
\hline & $R^{2}$ & $\Delta R^{2}$ & $F$ & $\Delta F$ & Sig. $\Delta F$ & Unstand. coeff. $(B)$ & Stand. coeff. $(\beta)$ & Sig. \\
\hline \multicolumn{9}{|l|}{ Age 2.5} \\
\hline (Constant) & & & & & & -2.19 & & 0.10 \\
\hline Sex & 0.00 & 0.00 & 0.23 & 0.23 & 0.63 & -0.01 & -0.01 & 0.96 \\
\hline Birth weight & 0.05 & 0.05 & 2.53 & 4.81 & 0.03 & 0.00 & 0.23 & 0.03 \\
\hline Fetal risk factors & 0.07 & 0.02 & 2.24 & 1.63 & 0.20 & -0.39 & -0.33 & 0.01 \\
\hline Maternal BMI & 0.13 & 0.06 & 3.32 & 6.19 & 0.01 & 0.05 & 0.30 & 0.03 \\
\hline Smoking & 0.13 & 0.01 & 2.76 & 0.58 & 0.45 & 0.06 & 0.09 & 0.41 \\
\hline Breastfeeding dur. & 0.14 & 0.00 & 2.38 & 0.52 & 0.47 & 0.00 & -0.01 & 0.96 \\
\hline Household SES & 0.14 & 0.00 & 2.08 & 0.41 & 0.52 & -0.01 & -0.08 & 0.45 \\
\hline Mat. gen. depr. & 0.14 & 0.00 & 1.80 & 0.00 & 0.96 & 0.00 & -0.02 & 0.86 \\
\hline Mat. life events & 0.15 & 0.00 & 1.65 & 0.50 & 0.48 & -0.04 & -0.08 & 0.50 \\
\hline Exposure timing & 0.15 & 0.01 & 1.53 & 0.57 & 0.45 & 0.00 & -0.07 & 0.49 \\
\hline Obj. hardship & 0.15 & 0.00 & 1.38 & 0.03 & 0.87 & -0.08 & -0.07 & 0.56 \\
\hline Subj. distress & 0.17 & 0.01 & 1.38 & 1.37 & 0.25 & 0.13 & 0.14 & 0.25 \\
\hline \multicolumn{9}{|l|}{ Age 4} \\
\hline (Constant) & & & & & & -3.24 & & 0.04 \\
\hline Sex & 0.00 & 0.00 & 0.06 & 0.06 & 0.81 & 0.01 & 0.01 & 0.96 \\
\hline Birth weight & 0.03 & 0.03 & 0.95 & 1.84 & 0.18 & 0.00 & 0.16 & 0.16 \\
\hline Fetal risk factors & 0.09 & 0.06 & 2.10 & 4.30 & 0.04 & -0.10 & -0.07 & 0.56 \\
\hline Maternal BMI & 0.30 & 0.21 & 6.76 & 18.95 & $<0.01$ & 0.11 & 0.67 & $<0.01$ \\
\hline Smoking & 0.30 & 0.00 & 5.32 & 0.00 & 0.99 & -0.02 & -0.02 & 0.90 \\
\hline Breastfeeding dur. & 0.31 & 0.01 & 4.50 & 0.60 & 0.44 & 0.03 & 0.19 & 0.14 \\
\hline Household SES & 0.31 & 0.00 & 3.84 & 0.19 & 0.66 & 0.00 & -0.02 & 0.85 \\
\hline Mat. gen. depr. & 0.31 & 0.00 & 3.31 & 0.03 & 0.86 & 0.00 & 0.03 & 0.83 \\
\hline Mat. life events & 0.32 & 0.01 & 2.96 & 0.45 & 0.50 & -0.04 & -0.07 & 0.57 \\
\hline Exposure timing & 0.36 & 0.04 & 3.17 & 3.74 & 0.06 & 0.00 & -0.17 & 0.13 \\
\hline Obj. hardship & 0.39 & 0.02 & 3.13 & 2.15 & 0.15 & -0.29 & -0.22 & 0.07 \\
\hline Subj. distress & 0.41 & 0.03 & 3.17 & 2.55 & 0.12 & 0.20 & 0.19 & 0.12 \\
\hline \multicolumn{9}{|l|}{ Difference } \\
\hline (Constant) & & & & & & 0.13 & & 0.92 \\
\hline Sex & 0.00 & 0.00 & 0.02 & 0.02 & 0.90 & 0.07 & 0.04 & 0.74 \\
\hline Birth weight & 0.01 & 0.01 & 0.26 & 0.50 & 0.48 & 0.00 & 0.01 & 0.93 \\
\hline Fetal risk factors & 0.13 & 0.12 & 3.18 & 8.97 & $<0.01$ & 0.26 & 0.25 & 0.09 \\
\hline Maternal BMI & 0.14 & 0.01 & 2.62 & 0.92 & 0.34 & 0.02 & 0.14 & 0.37 \\
\hline Smoking & 0.20 & 0.06 & 3.06 & 4.28 & 0.04 & 0.29 & 0.23 & 0.08 \\
\hline Breastfeeding dur. & 0.20 & 0.00 & 2.56 & 0.25 & 0.62 & 0.01 & 0.10 & 0.49 \\
\hline Household SES & 0.20 & 0.00 & 2.16 & 0.04 & 0.84 & 0.00 & 0.00 & 0.98 \\
\hline Mat. gen. depr. & 0.21 & 0.01 & 1.97 & 0.66 & 0.42 & -0.01 & -0.12 & 0.37 \\
\hline Mat. life events & 0.21 & 0.00 & 1.73 & 0.06 & 0.80 & 0.03 & 0.06 & 0.69 \\
\hline Exposure timing & 0.27 & 0.06 & 2.09 & 4.42 & 0.04 & 0.00 & -0.27 & 0.03 \\
\hline Objective hardship & 0.28 & 0.01 & 1.94 & 0.58 & 0.45 & 0.11 & 0.11 & 0.41 \\
\hline Subjective distress & 0.28 & 0.00 & 1.76 & 0.17 & 0.68 & -0.05 & -0.06 & 0.68 \\
\hline
\end{tabular}

Abbreviations: BMI: body mass index; Breastfeeding dur.: breastfeeding duration (months); SES: socioeconomic status; Mat.: maternal; Gen. depr.: general depression.

even when controlling for child BMI $Z$-score at age 2.5 (full results not shown).

\subsection{Central Adiposity (SS : TR)}

3.5.1. Age 2.5. There were no effects of covariates or of the severity of objective hardship or subjective distress due to the flood on central adiposity at age 2.5 at entry into the model.
In the final model, the fetal risk variable was the only predictor of central adiposity: fewer fetal risk factors predicted greater central adiposity $(P=0.04)$. There were no effects of the timing or severity of flood exposure.

3.5.2. Age 4. At entry into the model and in the final model, maternal general depression was the only predictor of central adiposity (at entry, $P=0.05$; final model, $P=0.04$ ). 
TABLE 3: Summary of hierarchical linear regression analyses for total adiposity (SS + TR). Significant effects are indicated in bold.

\begin{tabular}{|c|c|c|c|c|c|c|c|c|}
\hline \multirow{2}{*}{ Predictor variables } & \multicolumn{5}{|c|}{ Values at entry into model } & \multicolumn{3}{|c|}{ Final model } \\
\hline & $R^{2}$ & $\Delta R^{2}$ & $F$ & $\Delta F$ & Sig. $\Delta F$ & Unstand. coeff. $(B)$ & Stand. coeff. $(\beta)$ & Sig. \\
\hline \multicolumn{9}{|l|}{ Age 2.5} \\
\hline (Constant) & & & & & & 5.73 & & 0.15 \\
\hline Sex & 0.03 & 0.03 & 2.51 & 2.51 & 0.12 & 0.98 & 0.18 & 0.11 \\
\hline Birth weight & 0.04 & 0.01 & 1.67 & 0.83 & 0.36 & 0.00 & 0.14 & 0.21 \\
\hline Fetal risk factors & 0.05 & 0.01 & 1.47 & 1.07 & 0.30 & -0.58 & -0.19 & 0.18 \\
\hline Maternal BMI & 0.08 & 0.03 & 1.80 & 2.69 & 0.10 & 0.10 & 0.20 & 0.17 \\
\hline Smoking & 0.08 & 0.00 & 1.42 & 0.02 & 0.90 & -0.03 & -0.02 & 0.87 \\
\hline Breastfeeding dur. & 0.09 & 0.01 & 1.32 & 0.80 & 0.37 & -0.02 & -0.06 & 0.63 \\
\hline Household SES & 0.12 & 0.03 & 1.48 & 2.34 & 0.13 & 0.04 & 0.15 & 0.22 \\
\hline Mat. gen. depr. & 0.13 & 0.01 & 1.41 & 0.94 & 0.33 & 0.04 & 0.11 & 0.35 \\
\hline Mat. life events & 0.14 & 0.01 & 1.36 & 0.91 & 0.34 & -0.15 & -0.12 & 0.37 \\
\hline Exposure timing & 0.18 & 0.05 & 1.70 & 4.24 & 0.04 & -0.01 & -0.25 & 0.03 \\
\hline Objective hardship & 0.20 & 0.02 & 1.70 & 1.60 & 0.21 & 0.43 & 0.14 & 0.28 \\
\hline Subjective distress & 0.20 & 0.00 & 1.54 & 0.06 & 0.80 & 0.08 & 0.03 & 0.80 \\
\hline \multicolumn{9}{|l|}{ Age 4} \\
\hline (Constant) & & & & & & 9.13 & & 0.10 \\
\hline Sex & 0.00 & 0.00 & 0.02 & 0.02 & 0.90 & -0.21 & -0.03 & 0.82 \\
\hline Birth weight & 0.00 & 0.00 & 0.11 & 0.21 & 0.65 & 0.00 & 0.00 & 0.98 \\
\hline Fetal risk factors & 0.02 & 0.02 & 0.33 & 0.78 & 0.38 & -2.06 & -0.43 & 0.01 \\
\hline Maternal BMI & 0.24 & 0.22 & 3.78 & 13.85 & $<0.01$ & 0.31 & 0.61 & $<0.01$ \\
\hline Smoking & 0.47 & 0.23 & 8.29 & 20.27 & $<0.01$ & 4.80 & 0.45 & $<0.01$ \\
\hline Breastfeeding dur. & 0.47 & 0.00 & 6.79 & 0.09 & 0.76 & 0.03 & 0.06 & 0.67 \\
\hline Household SES & 0.47 & 0.00 & 5.70 & 0.02 & 0.89 & 0.00 & 0.01 & 0.94 \\
\hline Mat. gen. depr. & 0.47 & 0.00 & 4.89 & 0.05 & 0.82 & 0.01 & 0.01 & 0.94 \\
\hline Mat. life events & 0.47 & 0.00 & 4.28 & 0.15 & 0.70 & -0.16 & -0.07 & 0.60 \\
\hline Exposure timing & 0.48 & 0.01 & 3.92 & 0.84 & 0.37 & -0.01 & -0.11 & 0.36 \\
\hline Objective hardship & 0.49 & 0.00 & 3.52 & 0.22 & 0.64 & 0.02 & 0.00 & 0.97 \\
\hline Subjective distress & 0.52 & 0.03 & 3.57 & 2.63 & 0.11 & 0.73 & 0.21 & 0.11 \\
\hline \multicolumn{9}{|l|}{ Difference } \\
\hline (Constant) & & & & & & -1.15 & & 0.77 \\
\hline Sex & 0.02 & 0.02 & 0.99 & 0.98 & 0.33 & -0.12 & -0.02 & 0.86 \\
\hline Birth weight & 0.02 & 0.00 & 0.58 & 0.18 & 0.67 & 0.00 & 0.07 & 0.47 \\
\hline Fetal risk factors & 0.05 & 0.03 & 0.89 & 1.49 & 0.23 & -0.65 & -0.16 & 0.30 \\
\hline Maternal BMI & 0.10 & 0.05 & 1.29 & 2.42 & 0.13 & 0.07 & 0.14 & 0.33 \\
\hline Smoking & 0.54 & 0.44 & 10.74 & 43.84 & $<0.01$ & 5.41 & 0.60 & $<0.01$ \\
\hline Breastfeeding dur. & 0.54 & 0.00 & 8.78 & 0.06 & 0.80 & 0.05 & 0.11 & 0.34 \\
\hline Household SES & 0.54 & 0.00 & 7.38 & 0.07 & 0.79 & -0.01 & -0.02 & 0.83 \\
\hline Mat. gen. depr. & 0.55 & 0.01 & 6.45 & 0.52 & 0.47 & -0.08 & -0.19 & 0.07 \\
\hline Mat. life events & 0.57 & 0.03 & 6.28 & 2.77 & 0.10 & 0.44 & 0.23 & 0.04 \\
\hline Exposure timing & 0.58 & 0.00 & 5.55 & 0.15 & 0.70 & 0.00 & -0.08 & 0.42 \\
\hline Objective hardship & 0.63 & 0.06 & 6.20 & 5.96 & 0.02 & 0.94 & 0.23 & 0.03 \\
\hline Subjective distress & 0.67 & 0.04 & 6.54 & 4.45 & 0.04 & 0.71 & 0.23 & 0.04 \\
\hline
\end{tabular}

Abbreviations: BMI: body mass index; Breastfeeding dur.: breastfeeding duration (months); SES: socioeconomic status; Mat.: maternal; Gen. depr.: general depression.

There were no effects of the timing or severity of flood exposure. The effects of maternal depression remained significant in the final model $(P=0.05)$ even when controlling for BMI $Z$-scores at age 4 (full results not shown).

3.5.3. Difference between Ages 2.5 and 4. At entry into the model, birth weight $(P=0.05)$ and maternal general depression $(P=0.01)$ predicted the difference in central adiposity between ages 2.5 and 4 . There were no effects of the timing or severity of flood exposure. In the final model, greater maternal depression predicted greater central adiposity $(P=$ $0.02)$; birth weight did not retain significance $(P=0.16)$. The effects of maternal depression remained significant in the final model $(P=0.03)$ even when controlling for BMI $Z$ scores at age 2.5 (full results not shown).

There were no effects of interaction terms in any model (results not shown). In all analyses, variance inflation factors 
TABLE 4: Summary of hierarchical linear regression analyses for central adiposity (SS : TR). Significant effects are indicated in bold.

\begin{tabular}{|c|c|c|c|c|c|c|c|c|}
\hline \multirow{2}{*}{ Predictor variables } & \multicolumn{5}{|c|}{ Values at entry into model } & \multicolumn{3}{|c|}{ Final model } \\
\hline & $R^{2}$ & $\Delta R^{2}$ & $F$ & $\Delta F$ & Sig. $\Delta F$ & Unstand. coeff. $(B)$ & Stand. coeff. $(\beta)$ & Sig. \\
\hline \multicolumn{9}{|l|}{ Age 2.5} \\
\hline (Constant) & & & & & & 0.76 & & $<0.01$ \\
\hline Sex & 0.00 & 0.00 & 0.42 & 0.41 & 0.52 & 0.03 & 0.09 & 0.42 \\
\hline Birth weight & 0.00 & 0.00 & 0.21 & 0.00 & 0.96 & 0.00 & 0.02 & 0.83 \\
\hline Fetal risk factors & 0.04 & 0.04 & 1.22 & 3.24 & 0.08 & -0.06 & -0.30 & 0.04 \\
\hline Maternal BMI & 0.05 & 0.01 & 1.03 & 0.47 & 0.50 & 0.00 & 0.15 & 0.34 \\
\hline Smoking & 0.06 & 0.01 & 1.02 & 0.98 & 0.32 & 0.01 & 0.12 & 0.34 \\
\hline Breastfeeding dur. & 0.09 & 0.03 & 1.28 & 2.50 & 0.12 & 0.00 & 0.20 & 0.14 \\
\hline Household SES & 0.11 & 0.02 & 1.34 & 1.66 & 0.20 & 0.00 & -0.17 & 0.17 \\
\hline Mat. gen. depr. & 0.13 & 0.03 & 1.50 & 2.39 & 0.13 & 0.00 & -0.20 & 0.10 \\
\hline Mat. life events & 0.14 & 0.01 & 1.38 & 0.50 & 0.48 & 0.01 & 0.10 & 0.46 \\
\hline Exposure timing & 0.14 & 0.00 & 1.25 & 0.23 & 0.63 & 0.00 & -0.05 & 0.65 \\
\hline Objective hardship & 0.14 & 0.00 & 1.12 & 0.00 & 0.99 & 0.00 & 0.00 & 0.97 \\
\hline Subjective distress & 0.14 & 0.00 & 1.02 & 0.01 & 0.91 & 0.00 & -0.01 & 0.91 \\
\hline \multicolumn{9}{|l|}{ Age 4} \\
\hline (Constant) & & & & & & 0.00 & & 1.00 \\
\hline Sex & 0.01 & 0.01 & 0.72 & 0.72 & 0.40 & 0.04 & 0.08 & 0.60 \\
\hline Birth weight & 0.08 & 0.07 & 2.31 & 3.85 & 0.06 & 0.00 & 0.21 & 0.17 \\
\hline Fetal risk factors & 0.08 & 0.00 & 1.52 & 0.02 & 0.89 & -0.02 & -0.08 & 0.69 \\
\hline Maternal BMI & 0.09 & 0.01 & 1.21 & 0.36 & 0.55 & 0.00 & -0.01 & 0.96 \\
\hline Smoking & 0.11 & 0.02 & 1.20 & 1.14 & 0.29 & 0.08 & 0.11 & 0.50 \\
\hline Breastfeeding dur. & 0.12 & 0.01 & 1.08 & 0.55 & 0.46 & 0.00 & 0.14 & 0.46 \\
\hline Household SES & 0.13 & 0.00 & 0.93 & 0.14 & 0.71 & 0.00 & 0.01 & 0.95 \\
\hline Mat. gen. depr. & 0.20 & 0.07 & 1.36 & 3.93 & 0.05 & 0.01 & 0.33 & 0.04 \\
\hline Mat. life events & 0.20 & 0.00 & 1.20 & 0.13 & 0.72 & -0.01 & -0.06 & 0.71 \\
\hline Exposure timing & 0.23 & 0.03 & 1.29 & 1.85 & 0.18 & 0.00 & -0.19 & 0.22 \\
\hline Objective hardship & 0.24 & 0.00 & 1.17 & 0.23 & 0.63 & -0.02 & -0.06 & 0.72 \\
\hline Subjective distress & 0.24 & 0.00 & 1.06 & 0.13 & 0.72 & -0.01 & -0.06 & 0.72 \\
\hline \multicolumn{9}{|l|}{ Difference } \\
\hline (Constant) & & & & & & -0.60 & & 0.19 \\
\hline Sex & 0.00 & 0.00 & 0.08 & 0.08 & 0.78 & 0.02 & 0.03 & 0.81 \\
\hline Birth weight & 0.07 & 0.07 & 1.98 & 3.89 & 0.05 & 0.00 & 0.20 & 0.16 \\
\hline Fetal risk factors & 0.08 & 0.00 & 1.36 & 0.18 & 0.67 & 0.10 & 0.29 & 0.19 \\
\hline Maternal BMI & 0.11 & 0.03 & 1.48 & 1.79 & 0.19 & -0.01 & -0.24 & 0.26 \\
\hline Smoking & 0.11 & 0.00 & 1.16 & 0.00 & 1.00 & -0.12 & -0.16 & 0.35 \\
\hline Breastfeeding dur. & 0.12 & 0.00 & 0.98 & 0.15 & 0.70 & 0.01 & 0.15 & 0.36 \\
\hline Household SES & 0.12 & 0.00 & 0.82 & 0.01 & 0.94 & 0.00 & 0.05 & 0.73 \\
\hline Mat. gen. depr. & 0.24 & 0.12 & 1.68 & 6.94 & 0.01 & 0.01 & 0.37 & 0.02 \\
\hline Mat. life events & 0.25 & 0.01 & 1.54 & 0.53 & 0.47 & -0.02 & -0.12 & 0.45 \\
\hline Exposure timing & 0.30 & 0.06 & 1.80 & 3.36 & 0.07 & 0.00 & -0.27 & 0.07 \\
\hline Objective hardship & 0.31 & 0.00 & 1.61 & 0.16 & 0.69 & 0.01 & 0.03 & 0.82 \\
\hline Subjective distress & 0.33 & 0.03 & 1.64 & 1.61 & 0.21 & 0.05 & 0.20 & 0.21 \\
\hline
\end{tabular}

Abbreviations: BMI: body mass index; Breastfeeding dur.: breastfeeding duration (months); SES: socioeconomic status; Mat.: maternal; Gen. depr.: general depression.

(VIF) were low (less than 2.8) indicating that results were not affected by multicollinearity among variables.

\section{Discussion}

Our results indicate that exposure to a natural disaster during early gestation predicts greater total adiposity at age 2.5 and a greater increase in BMI $Z$-scores from age 2.5 to 4 . These results suggest that early pregnancy is a sensitive period for the effects of prenatal stress on childhood growth. Furthermore, prenatal objective hardship and subjective distress exposure significantly and independently predicted a greater increase in total adiposity from age 2.5 to 4 years; a greater number of stressful maternal life events (other 
than the flood) before and during pregnancy predicted this increase independently of the flood variables. Timing of flood exposure in pregnancy, objective hardship, and subjective distress together increased variance explained by up to $10 \%$ over and above that explained by covariates. This supports other studies suggesting that prenatal stress exposure can increase adiposity. Furthermore, our results highlight that effects are evident even in early childhood, which might be a particularly sensitive period for the development of obesity in adulthood [40].

As noted above, the effects of prenatal stress on later growth outcomes might reflect effects on central regulators of metabolism or metabolism at the cellular level, as well as through adverse effects on early growth [17]. The effects of stress exposure in our study persisted even after controlling for birth weight, which supports effects of prenatal stress on central regulators of growth and metabolism rather than through early growth patterns alone.

We observed no effects of flood exposure on central adiposity (SS:TR). However, maternal general depression predicted greater central adiposity at age 4 and a greater increase from age 2.5 to 4 . This supports results from Project Viva indicating that antenatal depression predicts greater central adiposity (SS : TR) at age 3 years [20], as well as studies indicating that greater maternal depressive symptoms predict greater risk of overweight in children aged 6-24 months [41]. Maternal depressive symptoms are often associated with adverse maternal health behaviors such as poor diet and exercise patterns, as well as adverse infant and child feeding patterns [41, 42].

Whereas our studies analyze depression at different time points and the mechanisms underlying the effects of prenatal depression are likely to differ from those of postpartum depression, they highlight the importance of maternal depression on adiposity in infancy and the need to distinguish between maternal stress, depression, anxiety, and other measures of maternal mental health in analyses. Differing physiological responses to stress, anxiety, and depression likely result in different mechanistic pathways underlying the effects of each factor on child outcomes [43]; a failure to distinguish between different measures of maternal mental health might obscure effects on child development.

4.1. Strengths and Limitations. Our study is limited by the relatively small sample size for some outcomes, which reduces statistical power and limits the analyses we can conduct. Furthermore, parental body size is a major predictor of children's body size. Although we were able to control for maternal BMI, we do not have anthropometric measurements for most of the children's fathers. However, since fathers' BMI is unlikely to be related to the timing or severity of flood exposure, it is unlikely that this introduces systematic bias into our analyses.

The independent nature of the stressor is the major strength of our study. Flood exposure is unlikely to be related to potentially confounding genetic or socioeconomic characteristics that might affect childhood body composition; for example, we found low correlation between objective hardship (IF100) and SES $(r<0.20)$ in the full sample. We were also able, unlike most studies, to tease apart the relative effects of maternal objective hardship and maternal distress to determine their relative effects. The prospective nature of the study is another strength. Our assessments included the measurement of many household and maternal characteristics that might act as confounders. The persistence of the effects of flood exposure, despite the inclusion of these covariates in all analyses, highlights that prenatal stress can independently affect body composition in childhood. Furthermore, these analyses extended results on the effects of maternal general depression on central adiposity, highlighting differences between the effects of maternal stress and maternal depression on childhood body composition and the need for further research.

\section{Conclusions}

Research on the developmental origins of health and disease, originally focused on poor maternal nutrition and later cardiometabolic diseases, now highlights that stress during pregnancy is also important in physical growth patterns and obesity risk [9]. Using the Iowa floods as a stressor, we show that exposure in early pregnancy and both objective and subjective stress are associated with greater adiposity in early childhood and a greater increase with age. With a strong body of the literature now supporting these relationships, we must begin to more precisely differentiate between effects of different aspects of maternal mental health on children's development. This research will complement mechanistic research on epigenetic pathways underlying the effects of maternal stress on children's development [44], with the ultimate goal of improving women's and children's health.

\section{Conflict of Interests}

The authors declare that there is no conflict of interests regarding the publication of this paper.

\section{Acknowledgments}

The authors wish to thank all of the families who have participated in the Iowa Flood Study. They are grateful to Jane Engeldinger for data collection and advice on obstetric history, Chunbo Yu for assistance with data analysis, and Corinne Hamlin, Bryan Koestner, and Christina Hao Zhang for data collection and entry. This study was supported by grants from the U.S. National Institute of Mental Health (NIMH) to Michael W. O'Hara, Suzanne King, Alain Brunet, Jane Engeldinger, David P. Laplante, Kimberly J. Hart, Cathy Vaillancourt, and Pathik Wadhwa and from the Canadian Institute of Health Research (CIHR) to Suzanne King, Michael W. O’Hara, Alain Brunet, Elysia Poggi Davis, David P. Laplante, Norbert Schmitz, and Cathy Vaillancourt. Kelsey N. Dancause was supported by fellowships from CIHR and the U.S. National Institutes of Health (NIH) while working on this paper. 


\section{References}

[1] J. Baird, D. Fisher, P. Lucas, J. Kleijnen, H. Roberts, and C. Law, "Being big or growing fast: systematic review of size and growth in infancy and later obesity," British Medical Journal, vol. 331, no. 7522, pp. 929-931, 2005.

[2] C. Corvalán, J. Kain, G. Weisstaub, and R. Uauy, "Impact of growth patterns and early diet on obesity and cardiovascular risk factors in young children from developing countries," Proceedings of the Nutrition Society, vol. 68, no. 3, pp. 327-337, 2009.

[3] N. Stettler, S. K. Kumanyika, S. H. Katz, B. S. Zemel, and V. A. Stallings, "Rapid weight gain during infancy and obesity in young adulthood in a cohort of African Americans," The American Journal of Clinical Nutrition, vol. 77, no. 6, pp. 13741378, 2003.

[4] L. Dubois and M. Girard, "Early determinants of overweight at 4.5 years in a population-based longitudinal study," International Journal of Obesity, vol. 30, no. 4, pp. 610-617, 2006.

[5] J. A. Yanovski, "Rapid weight gain during infancy as a predictor of adult obesity," The American Journal of Clinical Nutrition, vol. 77, no. 6, pp. 1350-1351, 2003.

[6] H. Beydoun and A. F. Saftlas, "Physical and mental health outcomes of prenatal maternal stress in human and animal studies: a review of recent evidence," Paediatric and Perinatal Epidemiology, vol. 22, no. 5, pp. 438-466, 2008.

[7] K. L. K. Tamashiro, C. E. Terrillion, J. Hyun, J. I. Koenig, and T. H. Moran, "Prenatal stress or high-fat diet increases susceptibility to diet-induced obesity in rat offspring," Diabetes, vol. 58, no. 5, pp. 1116-1125, 2009.

[8] S. Entringer, C. Buss, J. M. Swanson et al., "Fetal programming of body composition, obesity, and metabolic function: the role of intrauterine stress and stress biology," Journal of Nutrition and Metabolism, vol. 2012, Article ID 632548, 16 pages, 2012.

[9] S. Entringer, C. Buss, and P. D. Wadhwa, "Prenatal stress and developmental programming of human health and disease risk: concepts and integration of empirical findings," Current Opinion in Endocrinology, Diabetes and Obesity, vol. 17, no. 6, pp. 507-516, 2010.

[10] M. J. Lazinski, A. K. Shea, and M. Steiner, "Effects of maternal prenatal stress on offspring development: a commentary," Archives of Women's Mental Health, vol. 11, no. 5-6, pp. 363-375, 2008.

[11] C. Monk, J. Spicer, and F. A. Champagne, "Linking prenatal maternal adversity to developmental outcomes in infants: the role of epigenetic pathways," Development and Psychopathology, vol. 24, no. 4, pp. 1361-1376, 2012.

[12] L. Paternain, M. A. Batlle, A. L. De La Garza, F. I. Milagro, J. A. Martínez, and J. Campión, "Transcriptomic and epigenetic changes in the hypothalamus are involved in an increased susceptibility to a high-fat-sucrose diet in prenatally stressed female rats," Neuroendocrinology, vol. 96, no. 3, pp. 249-260, 2012.

[13] L. Cao-Lei, R. Massart, M. J. Suderman et al., "DNA methylation signatures triggered by prenatal maternal stress exposure to a natural disaster: Project Ice Storm," PLoS ONE, vol. 9, no. 9, Article ID e107653, 2014.

[14] J. G. Eriksson, "Early programming of later health and disease: factors acting during prenatal life might have lifelong consequences," Diabetes, vol. 59, no. 10, pp. 2349-2350, 2010.
[15] E. C. Cottrell and J. R. Seckl, "Prenatal stress, glucocorticoids and the programming of adult disease," Frontiers in Behavioral Neuroscience, vol. 3, article 19, 2009.

[16] J. E. Campbell, A. J. Peckett, A. M. D’Souza, T. J. Hawke, and M. C. Riddell, "Adipogenic and lipolytic effects of chronic glucocorticoid exposure," American Journal of Physiology: Cell Physiology, vol. 300, no. 1, pp. C198-C209, 2011.

[17] A. J. Drake, J. I. Tang, and M. J. Nyirenda, "Mechanisms underlying the role of glucocorticoids in the early life programming of adult disease," Clinical Science, vol. 113, no. 5-6, pp. 219-232, 2007.

[18] J. Li, J. Olsen, M. Vestergaard, C. Obel, J. L. Baker, and T. I. A. Sørensen, "Prenatal stress exposure related to maternal bereavement and risk of childhood overweight," PLoS ONE, vol. 5, no. 7, Article ID el1896, 2010.

[19] L. Hohwü, J. Li, J. Olsen, T. I. A. Sørensen, and C. Obel, "Severe maternal stress exposure due to bereavement before, during and after pregnancy and risk of overweight and obesity in young adult men: a danish national cohort study," PLOS ONE, vol. 9, no. 5, Article ID e97490, 2014.

[20] K. A. Ertel, K. C. Koenen, J. W. Rich-Edwards, and M. W. Gillman, "Antenatal and postpartum depressive symptoms are differentially associated with early childhood weight and adiposity," Paediatric and Perinatal Epidemiology, vol. 24, no. 2, pp. 179-189, 2010.

[21] M. W. Gillman, J. W. Rich-Edwards, S. Huh et al., "Maternal corticotropin-releasing hormone levels during pregnancy and offspring adiposity," Obesity, vol. 14, no. 9, pp. 1647-1653, 2006.

[22] K. G. Ingstrup, C. Schou Andersen, T. A. Ajslev, P. Pedersen, T. I. A. Sørensen, and E. A. Nohr, "Maternal distress during pregnancy and offspring childhood overweight," Journal of Obesity, vol. 2012, Article ID 462845, 7 pages, 2012.

[23] B. R. Mueller and T. L. Bale, "Impact of prenatal stress on long term body weight is dependent on timing and maternal sensitivity," Physiology and Behavior, vol. 88, no. 4-5, pp. 605614, 2006.

[24] L. M. Glynn, P. D. Wadhwa, C. Dunkel-Schetter, A. ChiczDeMet, and C. A. Sandman, "When stress happens matters: effects of earthquake timing on stress responsivity in pregnancy," American Journal of Obstetrics and Gynecology, vol. 184, no. 4, pp. 637-642, 2001.

[25] S. King, K. Dancause, A.-M. Turcotte-Tremblay, F. Veru, and D. P. Laplante, "Using natural disasters to study the effects of prenatal maternal stress on child health and development," Birth Defects Research Part C: Embryo Today: Reviews, vol. 96, no. 4, pp. 273-288, 2012.

[26] K. N. Dancause, D. P. Laplante, C. Oremus, S. Fraser, A. Brunet, and S. King, "Disaster-related prenatal maternal stress influences birth outcomes: Project Ice Storm," Early Human Development, vol. 87, no. 12, pp. 813-820, 2011.

[27] K. N. Dancause, D. P. Laplante, S. Fraser et al., "Prenatal exposure to a natural disaster increases risk for obesity in 5-year-old children," Pediatric Research, vol. 71, no. 1, pp. 126-131, 2012.

[28] K. N. Dancause, F. Veru, R. E. Andersen, D. P. Laplante, and S. King, "Prenatal stress due to a natural disaster predicts insulin secretion in adolescence," Early Human Development, vol. 89, no. 9, pp. 773-776, 2013.

[29] K. J. Nylen, M. W. O’Hara, and J. Engeldinger, “Perceived social support interacts with prenatal depression to predict birth outcomes," Journal of Behavioral Medicine, vol. 36, no. 4, pp. 427-440, 2013. 
[30] T. G. Lohman, A. F. Roche, and R. Martorell, Anthropometric Standardization Reference Manual, Human Kinetics Books, Champaign, Ill, USA, 1988.

[31] A. B. Hollingshead, Four-Factor Index of Social Status, Yale University Press, New Haven, Conn, USA, 1973.

[32] I. G. Sarason, J. H. Johnson, and J. M. Siegel, "Assessing the impact of life changes: development of the life experiences survey," Journal of Consulting and Clinical Psychology, vol. 46, no. 5, pp. 932-946, 1978.

[33] D. Watson, M. W. O’Hara, L. J. Simms et al., "Development and validation of the inventory of depression and anxiety symptoms (IDAS)," Psychological Assessment, vol. 19, no. 3, pp. 253-268, 2007.

[34] E. Bromet and M. A. Dew, "Review of psychiatric epidemiologic research on disasters," Epidemiologic Reviews, vol. 17, no. 1, pp. 113-119, 1995.

[35] A. C. McFarlane, "Relationship between psychiatric impairment and a natural disaster: the role of distress," Psychological Medicine, vol. 18, no. 1, pp. 129-139, 1988.

[36] R. L. Brock, M. W. O’Hara, K. J. Hart et al., "Partner support and maternal depression in the context of the Iowa floods," Journal of Family Psychology, vol. 28, no. 6, pp. 832-843, 2014.

[37] D. S. Weiss and C. R. Marmar, "The impact of event scalerevised," in Assessing Psychological Trauma and PTSD: A Practitioner's Handbook, J. P. Wilson and T. M. Keane, Eds., pp. 399411, Guilford, New York, NY, USA, 1997.

[38] CDC (Centers for Disease Control and Prevention), Growth Charts, Centers for Disease Control and Prevention, Atlanta, Ga, USA, 2010, http://www.cdc.gov/growthcharts/.

[39] M.-E. Mathieu and L. Béliveau, "The use of skinfolds in anthropometric measures and their applications to diabetes," in Handbook of Anthropometry: Physical Measures of Human Form in Health and Disease, V. R. Preedy, Ed., vol. 1, Springer, London, UK, 2012.

[40] R. C. Whitaker, M. S. Pepe, J. A. Wright, K. D. Seidel, and W. H. Dietz, "Early adiposity rebound and the risk of adult obesity," Pediatrics, vol. 101, no. 3, p. E5, 1998.

[41] P. J. Surkan, I. Kawachi, and K. E. Peterson, "Childhood overweight and maternal depressive symptoms," Journal of Epidemiology and Community Health, vol. 62, no. 5, p. ell, 2008.

[42] R. S. Gross, N. K. Velazco, R. D. Briggs, and A. D. Racine, "Maternal depressive symptoms and child obesity in lowincome urban families," Academic Pediatrics, vol. 13, no. 4, pp. 356-363, 2013.

[43] R. Graignic-Philippe, J. Dayan, S. Chokron, A.-Y. Jacquet, and S. Tordjman, "Effects of prenatal stress on fetal and child development: a critical literature review," Neuroscience and Biobehavioral Reviews, vol. 43, pp. 137-162, 2014.

[44] P. D. Wadhwa, C. Buss, S. Entringer, and J. M. Swanson, "Developmental origins of health and disease: brief history of the approach and current focus on epigenetic mechanisms," Seminars in Reproductive Medicine, vol. 27, no. 5, pp. 358-368, 2009. 


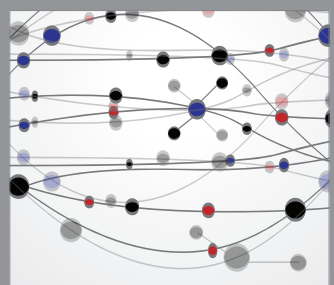

The Scientific World Journal
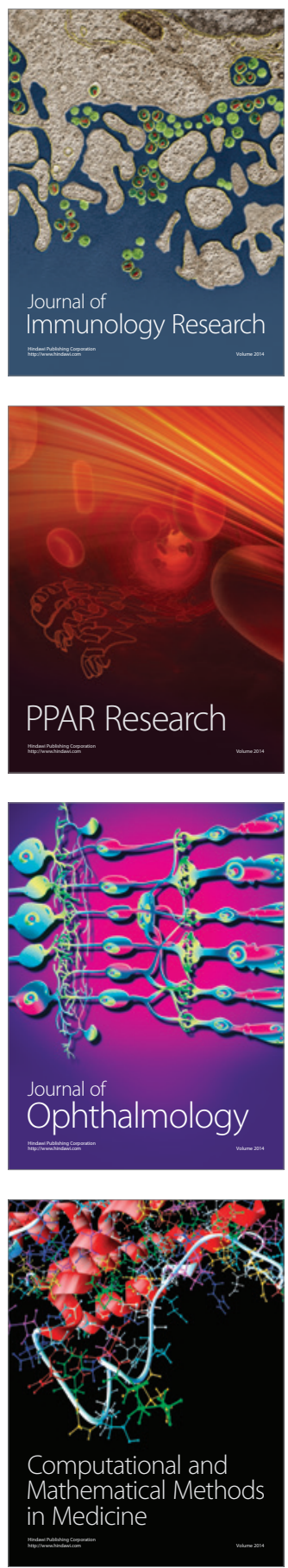

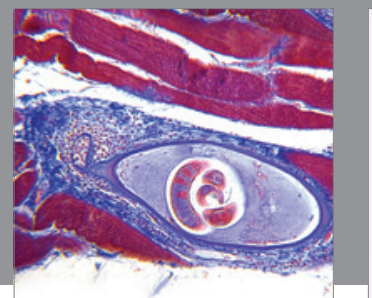

Gastroenterology

Research and Practice
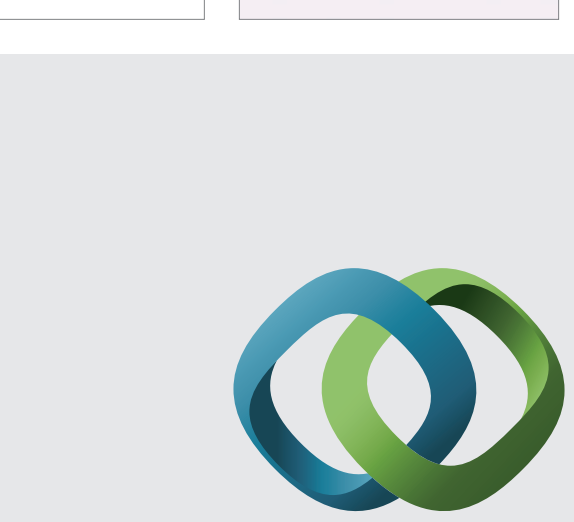

\section{Hindawi}

Submit your manuscripts at

http://www.hindawi.com
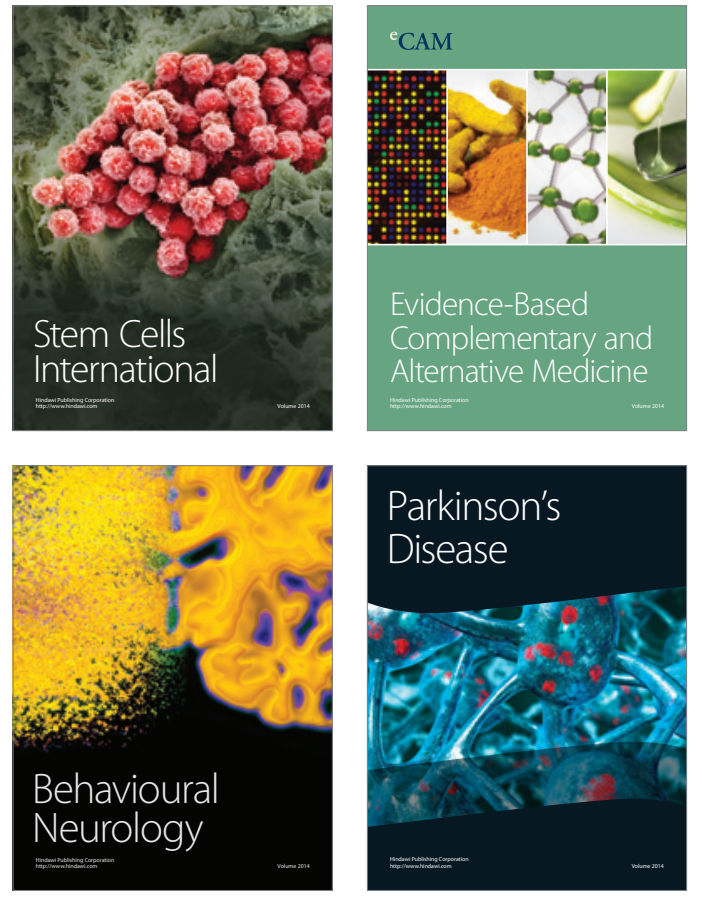
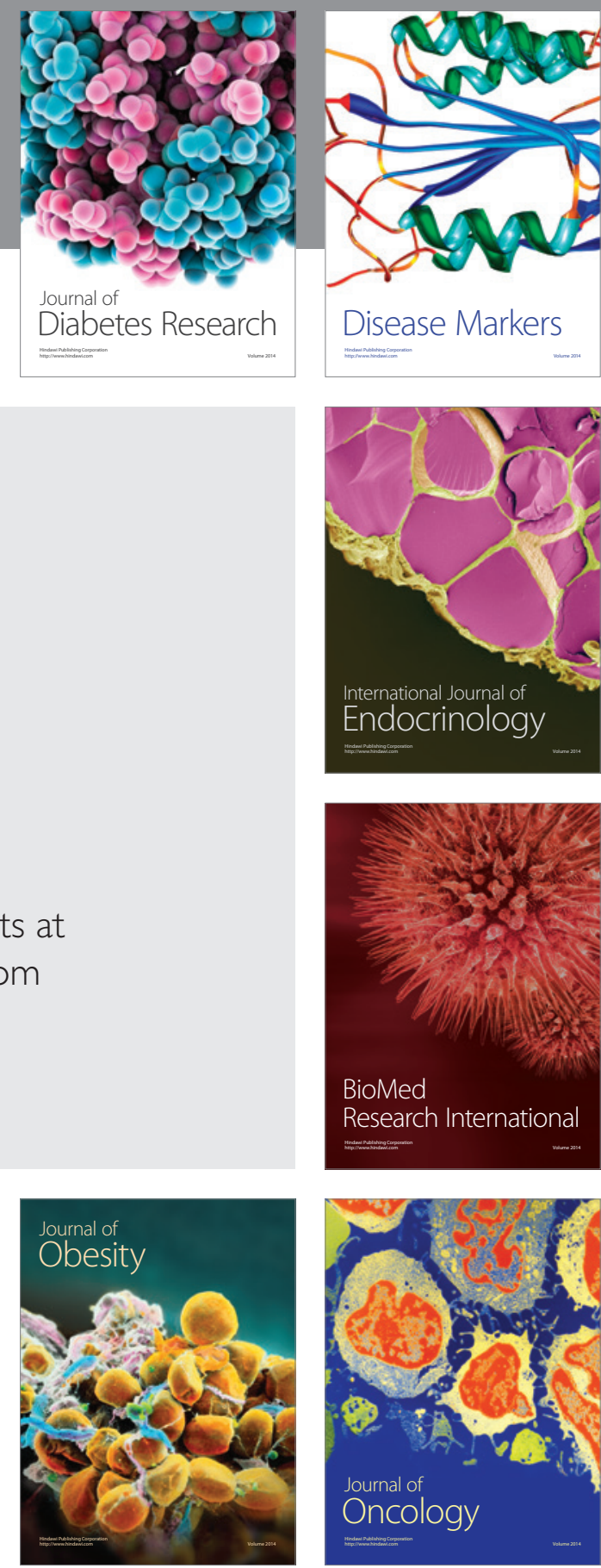

Disease Markers
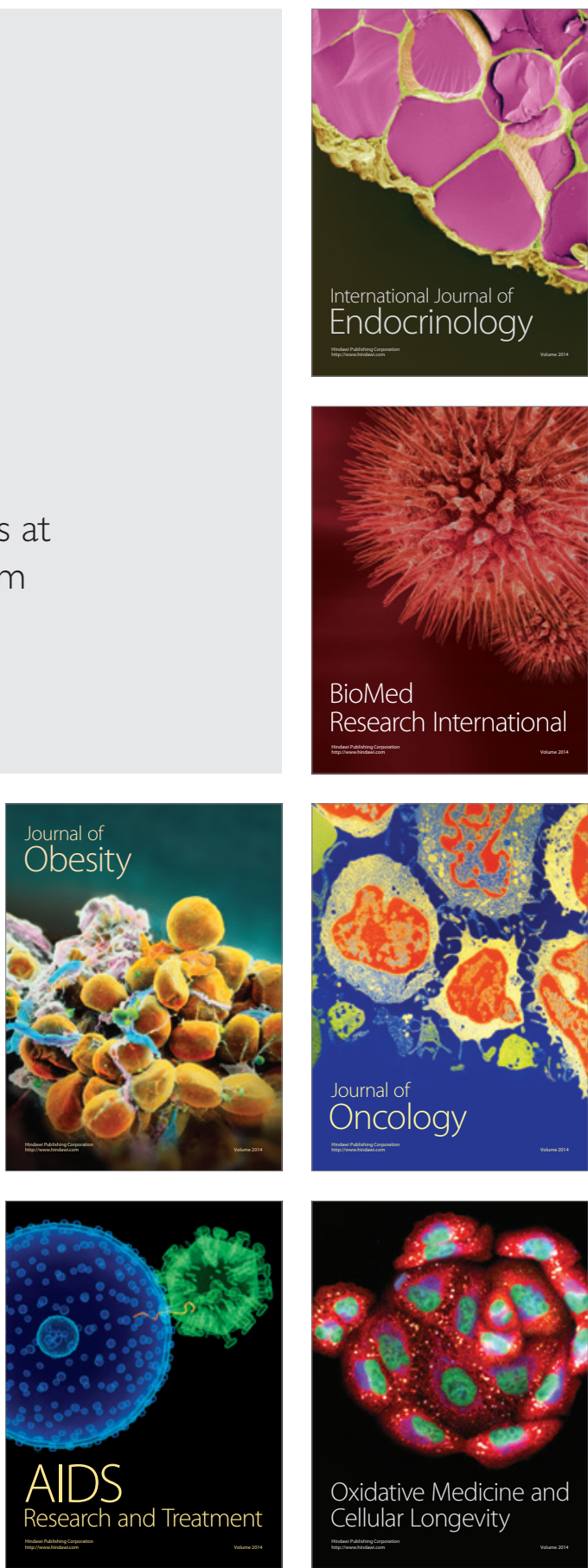